\title{
Une rareté sigillographique.
}

La Revue doit à l'obligeance de M. Giacomo Tropea, professeur à l'Université de Padoue, la communication de l'estampage reproduit ci-contre. Cet estampage a été pris sur un sceau de fer, "trouvé», nous écrit M. Tropea, "soit en Calabre (province de Reggio), soit dans la province de Messine ». ${ }^{1}$ ) A la partie supérieure du sceau est fixée une sorte d'anse ou d'anneau, où se trouve gravé un signe énigmatique, qui pourrait passer pour la représentation rudimentaire d'un arbre.

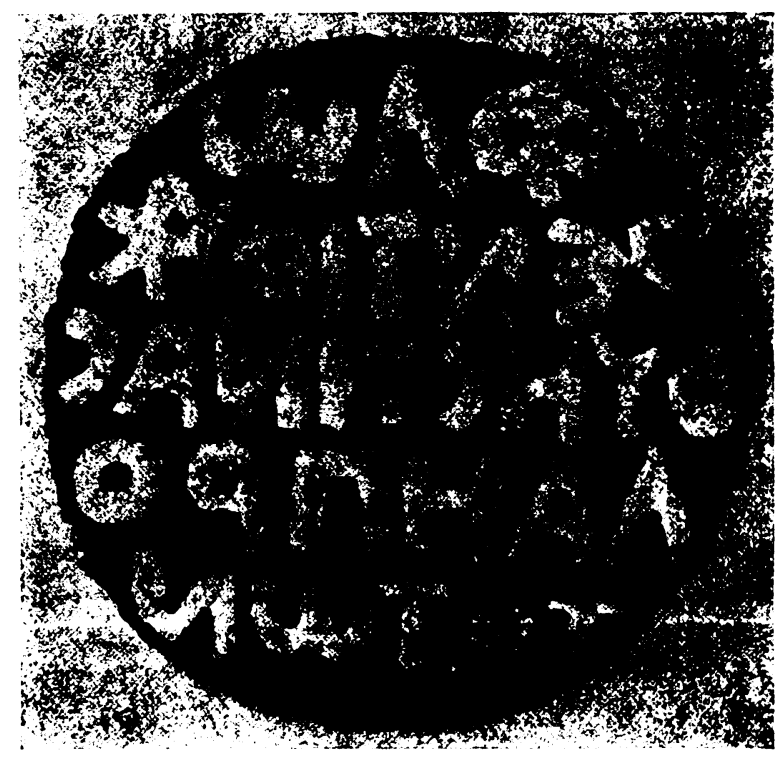

Les caractères de l'inscription sont en creux, non en relief, et se lisent de gauche à droite. Notre monument n'est donc pas une de ces bulles byzantines qu'on retrouve en si grand nombre, mais une matrice servant à en faire; ceci ressort avec évidence des trois circonstances que nous venons de signaler: le métal, l'anneau, les caractères gravés en creux.

1) Ce sceau est en la possession de M. Michele Trovato, via S. Pancrazio Giordini (Messina). 
Or, les matrices de sceaux byzantins sont excessivement rares. ${ }^{1}$ ) M. Schlumberger ne connaît pas un seul moule ayant servi à sceller en cire; quant aux matrices de molybdobulles, «à part un petit boullotirion en forme de pinces qu'il a vu à Athènes, chez M. Roussopoulo", il avoue "ne pouvoir citer d'autre exemple d'un de ces instruments». Notre moule appartient-il à la catégorie des $\beta o v \lambda \lambda \omega \tau \eta \dot{\rho} \iota \alpha$ proprement dits, ou servait-il simplement à faire des empreintes sur la cire? M. Schlumberger, je le sais, estime que cette dernière façon de sceller était presque inconnue des Byzantins. Cependant il ne manque point, dans la littérature, de passages où il en est fait mention. Je me borne à un exemple. Codinus, parlant de l'office du parakimômène $\tau \tilde{\eta} s \sigma \varphi \varepsilon \nu \delta o ́ v \eta s$, dit que ce dignitaire scelle au moyen de cire les lettres de l'Empereur à ses proches. ${ }^{2}$ ) Il me semble que la forme même du moule calabrais doit faire penser plutôt aux

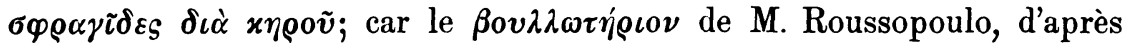
la description de M. Schlumberger, paraît être très différent. La disposition «en fer à gaufres» est nécessaire lorsqu'il s'agit d'un métal comme le plomb; pour la cire elle est inutile. On pourrait admettre, il est vrai, que notre moule n'est plus entier et que la moitié consistant en une plaque semblable à la nôtre, avec une inscription et peut-être une effigie, s'en est perdue. Mais cette hypothèse, comme nous le verrons, n'est ni nécessaire ni même particulièrement vraisemblable. L'anneau servait sans nul doute à suspendre le moule au cou de son propriétaire. $^{3}$ )

Examinons l'inscription. Je lis

\section{$\omega \wedge \Phi$ \\ XOITNЭT \\ JAИIT JHYO \\ OQTMA^ \\ UWTAT}

C'est à dire

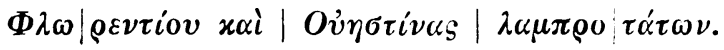

Cette lecture n'est pas absolument certaine. La ligature compliquée de la seconde ligne peut récéler autre chose que les lettres YKAI.

Néanmoins il est clair que l'inscription signifie: (sceau) de Florentius et de Vestina, $\lambda \alpha \mu \pi \rho o ́ \tau \alpha \tau o \iota$.

Le génitif se comprend sans qu'il faille supposer que l'inscription commençait ou se continuait sur la partie perdue du moule - l'autre

1) G. Scblumberger, S'igillographie de l'Empire byzantin p. 8 et 10.

2) Codini Curopalatae De officiis p. 34 ed. Bonn.

3) Schlumberger, op. cit. p. 10. 
face du sceau. M. Schlumberger, dans sa Sigillographie, donne de

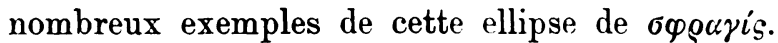

Les deux personnages doivent être deux époux. Car le génitif

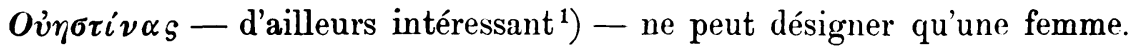
Nous n'avons pas trouvé un seul sceau associant ainsi les noms de deux époux. ${ }^{2}$ Florentius et Vestina sont certainement des Grees d' Italie. Le nom de Florentius, très fréquent en Italie, parait être rare $\mathfrak{a}$ Byzance. Le $\Phi \lambda \omega \varrho \varepsilon ́ v \tau \iota$ S d'après lequel Nicéphore Grégoras a intitulé son dialogue philosophique contre Barlaam est un personnage fictif.

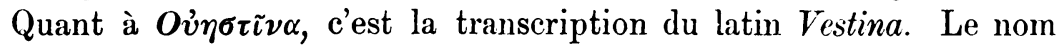
est bien connu, surtout sous sa forme masculine Vestinus. ${ }^{3}$ ) Il se

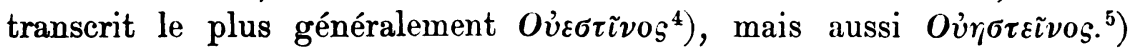
Vestina se rencontre une fois, sur une inscription trouvée dans le pays des Vestins ${ }^{6}$ ), où ce nom devait être surtout répandu. Peut-être notre Vestina était-elle de la même contrée que son homonyme de l'inscription latine.

Je ne dis rien de l'épithète $\lambda \alpha \mu \pi \rho o \tau \alpha ́ \tau \omega \nu$, si fréquente dans la titulature byzantine et qui accompagne toujours le nom de certains hauts fonctionnaires.

Est-il possible de dater approximativement notre inscription? Oui, car elle forme un de ces mauvais trimètres iambiques tels qu'ils apparaissent sur les bulles vers le milieu du $\mathrm{XI}^{e}$ siècle. ${ }^{7}$ ) Le sceau calabrais date donc probablement des derniers temps de la domination byzantine dans l'Italie méridionale.

Hu y (Belgique).

\section{Henri Grégoire.}

1) Cf. K. Dieterich, Untersuchungen z. Gesch. d. gr. Spr., p. 173: Eindringen des $\alpha$ impurum in den Gen. und Dat. Il est remarquable que ce phénomène apparaisse tout d'abord dans la déclinaison de mots étrangers, surtout latins. Les

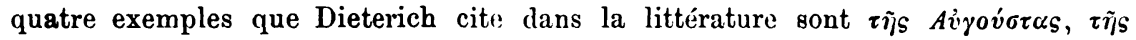
$\beta i \gamma \lambda \alpha \varsigma, \tau \tilde{\eta} \varsigma \sigma x \alpha^{\prime} \lambda \alpha_{\varsigma}, \tau \tilde{\eta} \tau \alpha^{\prime} \beta \lambda \alpha$ (Constantin Porphyrogénète). J'y ajouterai $\tau \tilde{\eta} s \sigma \tau \rho \alpha^{\prime} \tau \alpha$, dans les Miracula B. Mariae in Choziba, Anal. Boll. VII 363 1. 10 (texte du VII• siècle).

2) M. Schlumberger, dans la section de son livre consacré aux sceaux patronymiques, enregistre des sceaux où la femme du propriétaire est citée, mais uniquement pour désigner clairement celui-ci.

3) Cf. CIL IX, Index cognominum, et Prosopographia imperii romani, s. v.

4) Pape, s. v. 5) CIG III 4957, 28. 30. 6) CIL IX 3471.

7) Cf. Fröhner, Bulles métriques (dans l'Annuaire de la société française de numismatique et d'archéologie, 6 (1882), p. 40 et suiv.). La forme des caractères rappelle aussi les bulles de cette époque. 